\title{
LAS CONCEPCIONES DE LOS PROFESIONALES DE LA ENSEÑANZA SOBRE EL DESARROLLO Y EVALUACIÓN DE LAS COMPETENCIAS TRANSVERSALES EN EL GRADO DE MAGISTERIO EN EDUCACIÓN PRIMARIA DE LA FACULTAD DE EDUCACIÓN DE LA UNIVERSIDAD DE ZARAGOZA
}

THE CONCEPTIONS OF TEACHING PROFESSIONALS ON THE DEVELOPMENT AND EVALUATION OF TRANSVERSAL COMPETENCES DURING THE PRIMARY EDUCATION TEACHING DEGREE AT THE FACULTY OF EDUCATION IN THE UNIVERSITY OF ZARAGOZA

\section{LES CONCEPTIONS DES PROFESSIONNELS DE L'ENSEIGNEMENT SUR LE DÉVE- LOPPEMENT ET L'ÉVALUATION DES COMPÉTENCES TRANSVERSALES PENDANT LE DIPLÔME D'ENSEIGNEMENT DANS L'ÉDUCATION PRIMAIRE DE LA FACULTÉ D'ÉDUCATION DE L'UNIVERSITÉ DE SARAGOSSE}

Silvia Lorente-Echeverría, Carlos Peñarrubia-Lozano, Inmaculada Canales-Lacruz y Berta Murillo-Pardo Universidad de Zaragoza

\section{Resumen:}

Este trabajo presenta una evaluación sobre el modo en el que se están desarrollando y evaluando las competencias transversales en el Grado de Magisterio en Educación Primaria de la Facultad de Educación de la Universidad de Zaragoza. El análisis ofrece una perspectiva cualitativa, profundizando en las opiniones de 26 miembros de diferentes grupos de la comunidad educativa -estudiantes, profesores, y maestros en activo y jubilados-. La recogida de datos se efectuó mediante grupos de discusión, posteriormente analizados mediante el programa Nvivo11. Los principales resultados muestran la necesidad de poner en marcha un modelo por competencias en la Facultad de Educación, para poder evaluarlo y conocer el impacto que tiene en el modelo formativo del estudiante. Una constante reflexión crítica por parte del profesorado sobre su práctica docente y considerar la evaluación como un instrumento para conocer y descubrir el aprendizaje, son otras dos necesidades que se plantean en este trabajo, además de caminar hacia una cultura de colaboración y de una mayor formación del profesorado, donde las competencias adquieran la importancia y prioridad que merecen. La inteligencia emocional y aprender a aprender aparecen como propuestas más relevantes.

Palabras clave: : Enseñanza; aprendizaje transformador; cooperación educativa; enseñanza primaria; formación docente basada en competencias 


\begin{abstract}
This study analyses how transversal skills are developed and evaluated in the Primary Education Teaching degree at the University of Zaragoza. To this aim, 26 members of various groups of the educational community were surveyed, including university students, professors, active and retired teachers. Proposals about competence process in the Faculty and implementing the changes associated with convergence towards the EHEA under the most appropriate conditions have been showed. The opinions were associated collected through discussion groups that were analysed afterwards using the software NVIVO11. The most relevant results show the importance of establishing a competences-based education model in the Education Faculty, in order to evaluate it and its impact on the students formation. A constant critical reflection by teachers about their teaching practice and consider evaluation as an instrument to learn and discover learning, are two other needs that arise in this work. In addition, to move towards a culture of collaboration and greater teacher training, where competences acquire the importance and priority they deserve. Emotional intelligence and "learn how to learn" appear as more relevant proposals. The results obtained in this project provide a tool that allows the Faculty to improve and advance toward integral training models for the teachers of the future.
\end{abstract}

Keywords: Students teaching; transformative learning; educational cooperation; primary education; competency based teacher education.

\title{
Résumé
}

Ce document présente une évaluation de la manière dont les compétences transversales sont mises en œuvre et évaluées au diplôme d'enseignement de l'éducation primaire de la Faculté d'Éducation de l'Université de Saragosse. L'analyse offre une perspective qualitative, en se penchant sur les opinions de 26 membres de différents groupes de la communauté éducative -étudiants, enseignants, enseignants actifs et retraités-. La collecte des données a été réalisée par le biais de groupes de discussion, qui ont ensuite été analysés à l'aide du programme Nvivo11. Les principaux résultats montrent la nécessité de mettre en œuvre un modèle basé sur les compétences dans la faculté d'éducation afin de pouvoir l'évaluer et de connaître l'impact qu'il a sur le modèle formatif de l'étudiant. Une réflexion critique constante de la part du corps enseignant sur sa pratique pédagogique et la considération de l'évaluation comme un instrument de connaissance et de découverte de l'apprentissage sont deux autres besoins soulevés dans ce travail, en plus de l'évolution vers une culture de collaboration et une plus grande formation des enseignants, où les compétences acquièrent l'importance et la priorité qu'elles méritent. L'intelligence émotionnelle et apprendre à apprendre apparaissent comme les propositions les plus pertinentes.

Mots-clés: Enseignement; apprentissage transformateur; coopération éducative; enseignement primaire; formation des enseignants basée sur les compétences.. 


\section{INTRODUCCIÓN}

El sistema educativo tiene como función principal preparar y formar a ciudadanos para una sociedad cambiante, incierta y compleja (López et al., 2018; Morales y Morales, 2018). Se aspira a que la formación universitaria pueda dar respuesta a esta situación, permitiendo en los estudiantes la adquisición de un aprendizaje competencial más cercano y acorde al momento en el que nos encontramos. Algunos autores (Fraile, 2006; González et al., 2018; Martínez y González, 2019) coinciden en que el Espacio Europeo de Educación Superior (EEES) necesita un escenario distinto en la formación universitaria, un modelo mejorado que nos lleve hacia un aprendizaje de calidad.

El cambio se concreta en la consecución de un aprendizaje autónomo y significativo, a través del cual el estudiante pueda desarrollarse personal y profesionalmente, tanto en el ámbito formal como en el no formal (Astigarraga y Carrera, 2018; Bicocca, 2018; Ibarra-Sáiz y Rodríguez-Gómez, 2020). En este sentido, el principal agente de cambio tiene que ser la universidad, la cual, debe de proporcionar soluciones a los problemas de la sociedad, y además otorgar a los ciudadanos recursos para emprender el cambio (Aguado et al., 2017; Benayas y Marcén, 2019).

En estos momentos, con la actual Agenda 2030 y los Objetivos de Desarrollo Sostenible (ODS) (UNESCO, 2017), la universidad tiene la oportunidad de desarrollar su propia agenda, de darle voz y ayudar a que los objetivos que se proponen se cumplan, y hacer que la sociedad camine hacia ese desarrollo sostenible. Integrar esta agenda en su día a día permitiría cambiar el modelo universitario existente, la formación y la calidad de la misma. Aportaría al alumnado la adquisición de aquellas competencias transversales del futuro ciudadano, demandadas por la propia sociedad y la agenda. Se deben crear estudiantes críticos, creativos, comprometidos, responsables y cívicos (Onieva, 2018). La formación inicial en la universidad es clave para la construcción de la identidad profesional, se trata de la primera etapa formal en el camino para ser maestro/a (Jarauta y Pérez, 2017). Posee el papel más importante para poder llegar a estos objetivos, pero se necesitan personas con cultura de 
cambio, aire fresco, personas comprometidas con el cambio y la sociedad del futuro.

A partir de esta necesidad de cambio, se ha reabierto el debate acerca del papel de las competencias en la formación de los estudiantes universitarios. Las características que posee la educación universitaria y el contexto en el que se desarrolla, reclaman una mayor profundización en el enfoque competencial, como base para ejercer la actividad docente (Aguado et al., 2017; Zabalza, 2012). En estos momentos, la calidad de la enseñanza universitaria se aleja de los conocimientos, los cuales son accesibles mediante la tecnología, y se enfoca hacia la planificación de unos objetivos que cubran las necesidades y posibilidades del alumnado (Jiménez et al., 2019).

Por lo tanto, uno de los conceptos más importantes que se introducen es el de competencia. En los objetivos de Bolonia quedaba definida como "Pericia, aptitud, idoneidad para hacer algo o intervenir en un asunto determinado" (Montero, 2010, p. 27). Se comienza a pensar en el aprendizaje a lo largo de la vida, buscando esencialmente ser capaz de adaptarnos a las demandas de la sociedad del conocimiento (Pinos, 2016). La UNESCO (2017, p. 1) destaca que "toda persona, en cualquier etapa de su vida, debe disponer de oportunidades de aprendizaje permanentes, a fin de adquirir los conocimientos y las competencias necesarias para hacer realidad sus aspiraciones y contribuir a la sociedad". Por lo tanto, la universidad no tiene que ser solo una fuente de conocimientos, sino también de competencias que permita a los estudiantes seguir aprendiendo en el futuro de forma autónoma (González et al., 2018). Estas últimas ideas conforman la importancia de analizar las competencias transversales como base para el aprendizaje para toda la vida, por su influencia en el desarrollo académico, profesional, personal e interpersonal (Bolívar, 2011; Fernández-Ferrer y Forés, 2016). Por todo ello, el presente estudio centra su análisis e investigación sobre dichas competencias transversales.

En este contexto de convergencia de los sistemas de educación superior se produce un cambio de paradigma en el modo de entender la educación. Autores como Ramos (2005) aseguran que para conseguir modelos de formación integral de los futuros maestros es necesaria una reflexión crítica, sistemática y organizada 
que propicie en el profesorado universitario el cuestionamiento sobre su desempeño, sus modos de enseñar y su relación con los alumnos.

Con todo ello, la formación de competencias requiere una rebelión cultural para pasar de una educación centrada en la enseñanza a una nueva educación basada en el aprendizaje. Para conseguir alcanzar una revolución cultural surge la necesidad de evolucionar y de implementar cambios y transformaciones asociados a la convergencia hacia el EESS en las condiciones más adecuadas (Azorín y Arnaiz, 2018). Todos estos cambios vistos como un universo en constante movimiento, complejo y difícil pero no imposible de conocer, dominar y vivir en armonía. Pero como destacan (Montero et al., 2017) tenemos que indagar en los procesos y resultados que están sucediendo en los nuevos grados universitarios.

Teniendo en cuenta nuestro contexto, el grado en Educación Primaria de la Facultad de Educación -Universidad de Zaragoza-, según la Orden ECl/3857/2007, de diciembre (BOE de 29 de diciembre 2007), los estudiantes deberán adquirir las siguientes competencias transversales: (CT1) Integrar las competencias de las diferentes materias, para orientar el Trabajo de Fin de Grado y poder aplicar los conocimientos a la práctica profesional. (CT 2) Entender el aprendizaje como un hecho global, complejo y transcendente, diseñando, planificando, organizando y animando situaciones de aprendizaje. (CT 3). Gestionar y autorregular la progresión de los aprendizajes adaptándose a nuevas situaciones e interrelacionando saberes para elaborar otros nuevos. (CT 4). Atender a la singularidad de los diferentes contextos profesionales. (CT 5). Implicar al alumnado en su aprendizaje y en su trabajo. (CT 6). Trabajar en equipo siendo capaz de ejercer diferentes roles dentro del grupo. (CT 7). Participar en la gestión institucional y la relación con su entorno social. (CT 8). Informar e implicar a la sociedad en los fines de la institución. (CT 9). Utilizar y aplicar las Tecnologías de la Información y la Comunicación (TIC), para aprender, comunicarse y compartir conocimientos en diferentes contextos. (CT 10). Desarrollar la capacidad de comunicar, para enseñar en la propia lengua, y en otra u otras lenguas europeas. (CT 11) Afrontar los deberes y los dilemas éticos de la profesión. (CT 12). Organizar lo 
propia formación continua y motivar la mejora de la calidad. (CT 13). Investigar sobre la propia práctica, introduciendo propuestas de innovación encaminadas a la mejora y generando ideas nuevas. (CT 14). Buscar, gestionar, procesar, analizar y comunicar la información de manera eficaz, crítica y creativa.

Todas las competencias transversales aparecen en la planificación de las enseñanzas de las diferentes áreas de conocimiento. Pero no hay una estructura de coordinación que permita el desarrollo y la evaluación de las competencias, y también establecer un seguimiento de los resultados a medio y largo plazo. Se llevan a cabo iniciativas y actividades en la adquisición de competencias genéricas y transversales dentro del Plan de Orientación Universitaria. Y se siguen poniendo en marcha procesos que buscan ajustar, tanto los resultados de aprendizaje de las asignaturas como la carga de trabajo, a través de proyectos de coordinación docente -Memoria de verificación vigente, modificada el 4 de mayo de 2020, Informe de evaluación de la calidad y de los resultados de aprendizaje-.

\section{OBJETIVOS}

Los objetivos generales del presente estudio son:

- Analizar la detección de problemas percibidas del modelo competencial desarrollado en el Grado de Magisterio de Primaria.

- Analizar las propuestas de mejora percibidas del modelo competencial desarrollado en el Grado de Magisterio de Primaria.

Los objetivos específicos son:

- Identificar los elementos de calidad que configuran el modelo competencial aplicado en el Grado de Magisterio de Primaria.

- Identificar los problemas de aplicación del modelo competencial.

- Identificar los elementos de calidad que tendría que configurar el modelo competencial.

- Identificar las estrategias de actuación que tendrían que aplicarse en el modelo competencial. 


\section{MÉTODO}

El estudio se ha llevado a cabo mediante un diseño transversal, con metodología cualitativa fundamentada en el análisis de contenido.

\section{Muestra}

Han participado 26 personas, seleccionadas mediante un muestreo intencional y no aleatorio, garantizando una representación de participantes masculinos y femeninos en cada grupo (Tabla 1). Los estudiantes fueron alumnos de cuarto curso del Grado en Magisterio en Educación Primaria de la Facultad de Educación, con participantes de las diferentes menciones ofertadas -Audición y lenguaje, Pedagogía Terapéutica, Lengua Francesa, Lengua Inglesa, Educación Musical y Educación Física-. Los maestros en activo pertenecían a diferentes colegios de Educación Primaria dentro de la Comunidad Autónoma de Aragón. El tercer grupo lo formaron maestros jubilados, con una media de 35 años de experiencia docente en centros educativos de Educación Primaria e, incluso, en la propia universidad de Zaragoza. El último grupo se componía de profesores de diferentes departamentos de la Facultad de Educación con docencia en el grado de Educación Primaria -Universidad de Zaragoza-.

Tabla 1

Participantes del estudio

\begin{tabular}{lccc} 
Grupo & Hombre & Mujeres & Total \\
\hline & $\mathrm{N}$ (edad media) & $\mathrm{N}($ edad media) & $\mathrm{N}$ (edad media) \\
\cline { 2 - 4 } Estudiantes & $3(23.6 \pm 3.05)$ & $6(21.3 \pm 0.51)$ & $9(22.1 \pm 1.96)$ \\
Profesores universitarios & $1(43 \pm 0.00)$ & $3(42.3 \pm 5.50)$ & $4(42.5 \pm 4.50)$ \\
Maestros en activo & $1(38 \pm 0.00)$ & $5(45.2 \pm 6.53)$ & $6(44.0 \pm 6.54)$ \\
Maestros jubilados & $6(66.6 \pm 2.65)$ & $1(64 \pm 0.00)$ & $7(66.2 \pm 2.62)$ \\
Total & 11 & 15 & 26 \\
Fuente: Elaboración propia. & & &
\end{tabular}


Todos los informantes fueron conocedores de los objetivos del estudio y dieron su consentimiento para la grabación en audio de sus intervenciones. Una vez finalizado el estudio, se hizo entrega a todos los participantes de un informe con los principales resultados obtenidos.

\section{Instrumentos}

La recogida de la información se realizó mediante grupos de discusión. Para su aplicación se utilizó un guión semi-estructurado, fundamentado en el modelo de evaluación de Stufflebeam CIPP -Contexto, Entrada, Proceso y Producto- (Stufflebeam y Shinkfield, 1987). Contaba con cuatro centros de interés: a) evaluar el contexto; por ejemplo: ¿Cómo consideráis el desarrollo y evaluación de competencias en la enseñanza superior, para la formación universitaria del grado en Educación Primaria? ¿Positivo o negativo? ¿En qué sentido?; b) ¿Crees que el marco o programa que existe ahora tiene las características que debería tener un modelo competencial?; c) ¿Cómo debería aplicarse el desarrollo y evaluación de las competencias transversales en el Grado?; y d) ¿Cómo valorarías globalmente los resultados del Grado en EP en relación a las competencias transversales?

Posteriormente se aplicó el análisis de contenido con el apoyo del programa QRS Nvivo 11. Para codificar la información aportada por los participantes se desarrolló un sistema de categorías mediante un proceso mixto inductivo y deductivo. En la Tabla 2 se presentan todas las categorías finalmente analizadas. 
Tabla 2

Sistema de categorías

Dimensión

Categoría

Indicador

1. Detección de problemas

1.1. Elementos de calidad

1.1.1. Identificación de los elementos de calidad

1.1.2. Prioridad de competencias transversales

1.2. Implementación

1.2.1. Problemas en la aplicación

2. Propuestas de mejora

2.1. Elementos de calidad

2.1.1. Identificación de propuestas de mejora

2.1.2. Propuesta de competencias transversales

2.2. Implementación

2.2.1. Aplicación de propuesta de mejora

2.2.2. Secuenciación

Fuente: Elaboración propia

Este sistema de categorías identifica la detección de problemas y propuestas de mejora como dimensiones del problema -objeto de estudio-. Hay dos categorías en cada dimensión, los elementos de calidad y la implementación. Por último, cada categoría tiene uno o varios indicadores que son el último nivel de concreción.

\section{Procedimiento}

La duración de cada uno de los cuatro grupos de discusión osciló entre los 45 y los 60 minutos. Posteriormente se transcribieron las intervenciones para llevar a cabo las tres fases propuestas por Bardin (2002): preanálisis, explotación del material y tratamiento e interpretación de datos.

- En la fase preanálisis se elaboró el sistema de categorías. En primer lugar se llevó a cabo una lectura superficial de las transcripciones y se diseñó un borrador. Posteriormente, se aplicó una prueba piloto sobre uno de los grupos seleccionada al azar. Aquellos indicadores que recibieron cero referencias fueron eliminados del sistema. Fue el caso de dos de los indicadores -secuenciación en la categoría implementación y rendimiento en la categoría de producto-que pertenecían a la dimensión 1 Detección de problemas. 
Tras la prueba piloto se aplicó un test de concordancia en un cálculo de acuerdo entre dos codificadoras independientes. Para ello, las codificadoras se sujetaron a un entrenamiento para alcanzar la máxima precisión y coherencia. Tuvieron acceso al sistema de categorías con la explicación detallada de cada dimensión, categoría e indicador. Se utilizó el coeficiente de kappa de Cohen como medida de fiabilidad, teniendo muy buena concordancia ( $k=.898)$.

- En la explotación de material se codificaron todas las transcripciones de forma consensuada por el equipo de investigación, previa selección de los fragmentos a analizar.

- El tratamiento e interpretación de los resultados se llevó a cabo utilizando el software para diseños cualitativos QRS Nvivo 11.

\section{RESULTADOS}

Se han codificado 172 referencias, de las cuales 73 (42\%) están relacionadas con la dimensión 1. Detección de problemas y las otras 99 (58\%), con la dimensión 2. Propuestas de mejora. En la Figura 1 se detalla el número de referencias por indicador.

\section{Figura 1}

Número de referencias por indicador

1.1.1. Identificación de los elementos de calidad

1.1.2. Prioridad de competencias transversales

1.2.1. Problemas en la aplicación

2.1.1. Identificación de propuestas de mejora

2.1.2. Propuesta de competencias transversales

2.2.1. Aplicación de propuesta de mejora

2.2.2. Secuenciación

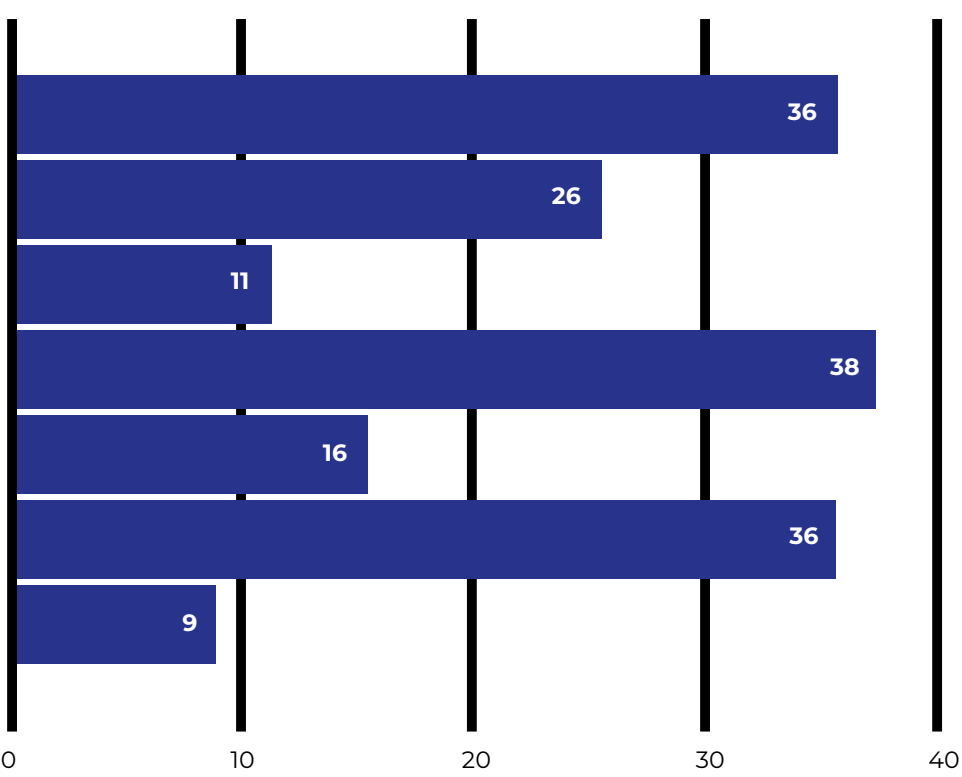

Fuente: elaboración propia. 
A continuación, se presenta el análisis interpretativo de los resultados. Las autorías de los fragmentos textuales que los acompañan son ficticias, asegurando con ello el anonimato de los informantes.

\section{Detección de problemas del modelo por competencias}

Esta dimensión tiene dos categorías: La categoría 1.1. Elementos de calidad y la 1.2. Implementación. A su vez, la primera categoría está formada por dos indicadores: Identificación elementos calidad -indicador 111-; Prioridad competencias transversales -indicador 112-; mientras que la segunda queda definida por el indicador Problemas de aplicación -indicador 121-. En la Figura 2 se detallan las principales ideas extraídas de los tres indicadores.

\section{Figura 2}

Mapa de la dimensión Detección de problemas

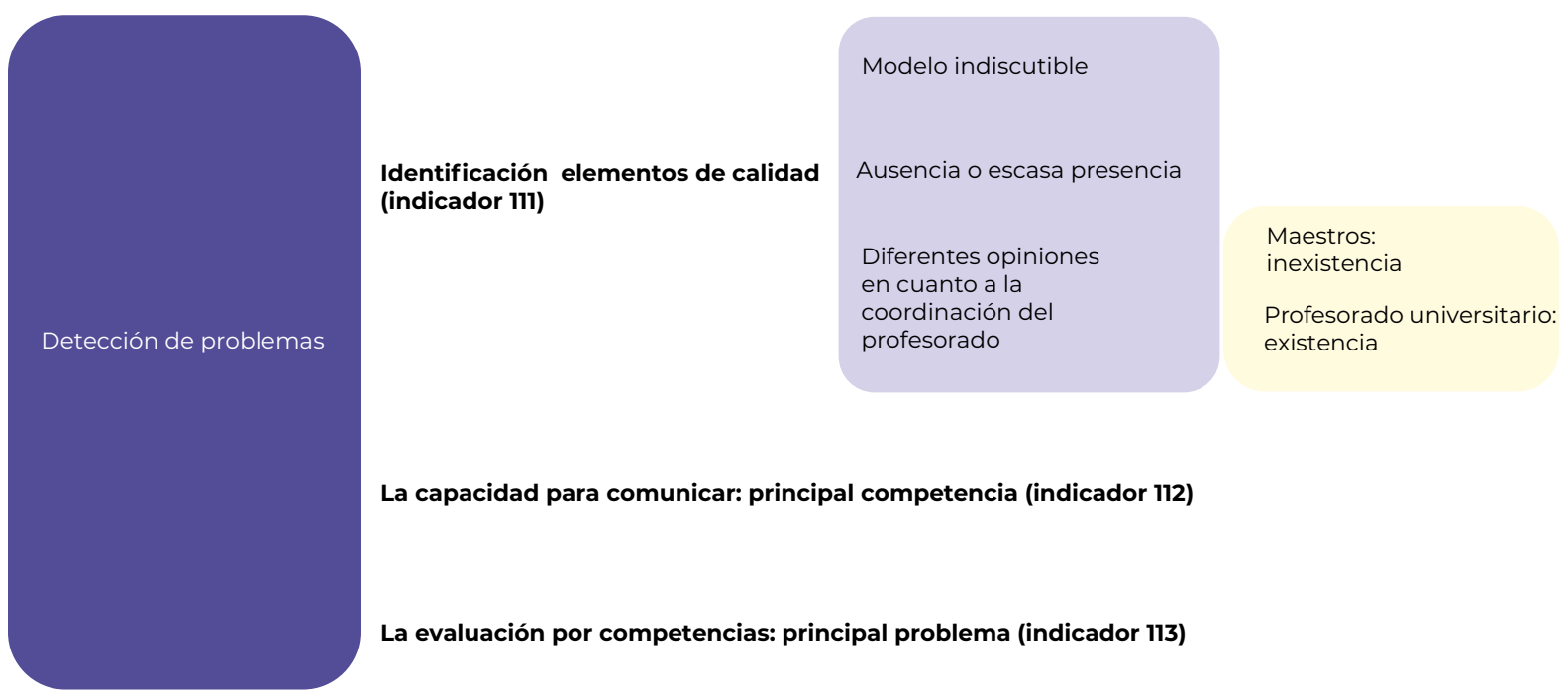

Fuente: elaboración propia.

El indicador Identificación elementos calidad -indicador 111-se refiere a la detección de los elementos que configuran el modelo por competencias. Con 14 referencias repartidas entre los cuatro grupos (38\% del total del indicador) los participantes han identificado como indispensable el modelo por competencias para trabajar en educación:

Yo pienso que lo bueno que tiene el trabajar por competencias es que vas directamente a las aptitudes que son necesarias. (...) son herramientas que un buen profesor debería tener y 
pienso que gracias al trabajo por competencias vas directamente a por ellas. (Darío, alumno).

La ausencia o escasa presencia de este modelo tiene ocho referencias (19\%). Lo más relevante son las seis pertenecientes al alumnado que exponen la rotunda ausencia del modelo en el sistema universitario: Yo creo que en general no lo hacen, que igual lo dicen pero en la práctica no se hace (Irene, alumna).

Con respecto a la perspectiva que tiene el profesorado hay ocho referencias, cinco de ellas procedentes del grupo de maestros -en activo y jubilados- que la describen como sesgada, en la que consideran que no existe coordinación del profesorado. En oposición, tres referencias procedentes del profesorado universitario afirman que: nos hemos coordinado, participado, aprendido los unos de los otros, etc. (Luisa, profesora universitaria).

El indicador 112 Prioridad competencias transversales pretendía analizar la opinión sobre la preponderancia de alguna de las 14 competencias. Nueve de 26 referencias codificadas en este indicador (37.5\%) han determinado la competencia transversal 10 -capacidad para comunicar-como la más relevante: El tema de las habilidades comunicativas pienso que es básico para cualquier persona que trabaja de cara el público, y más para un profesor que tienen que intentar transmitir unos conocimientos (Juan, maestro jubilado).

El indicador 121 Problemas en la aplicación tiene 11 referencias, y ocho de ellas establecen la dificultad que conlleva la evaluación de las competencias transversales en el Grado de Magisterio en educación primaria. Algunos de los puntos en los que los participantes encuentran dificultad son el diseño de instrumentos de evaluación, ya que, se hace muy difícil y muy difuso (Daniel, maestro en activo), y sobre todo la dificultad entraña en cómo o en qué momento de las diferentes acciones podemos llevar a cabo dicha evaluación (Blanca, profesora universitaria).

\section{Propuestas de mejora}

Esta dimensión tiene cuatro indicadores correspondientes a dos categorías, 2.1. Elementos de calidad y 2.2. Implementación respectivamente: Identificación de las propuestas -indicador 211-; Propuestas de competencias transversales -indica- 
dor 212-; Aplicación de propuestas -indicador 221-; y Secuenciación de competencias -indicador 222-. En la Figura 3 se detallan las principales ideas extraídas de los cuatro indicadores.

Figura 3

Mapa de la dimensión Propuestas de mejora

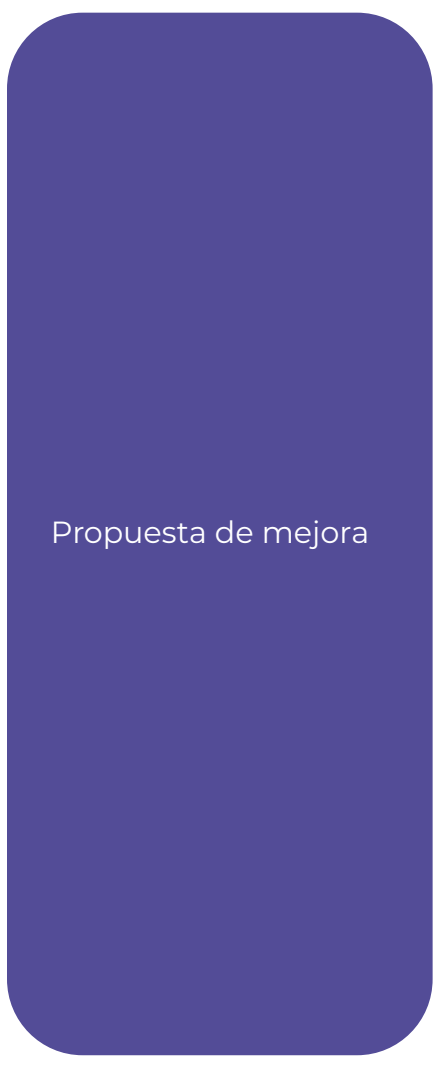

\section{Identificación propuestas (indicador 211)}

Propuestas de competencia (indicador 212)

\section{Aplicación de propuestas} (indicador 221)

Secuencias de competencias (indicador 222)
Trabajo en equipo

Compromiso de profesorado

Nuevo paradigma:

necesidades del alumnado

Inteligencia emocional

Aprender a aprender

Vínculo colegios-universidades

Concretar competencias

Trabajos interdisciplinares

Aumento número horas prácticas escolares

Diversidad de opiniones

Fuente: elaboración propia.

En el indicador Identificación propuestas -211- se han incluido todas las fórmulas para mejorar el modelo competencial establecidas por los participantes. Hay 38 referencias codificadas y 14 (37\% del indicador, véase Figura 3) destacan como principal propuesta de mejora el trabajo en equipo que requiere este modelo. Luis (alumno) expone: Porque yo considero que para que nos enseñen a trabajar en equipo primero deben comenzar a colaborar y cooperar entre ellos para poder transmitir estas competencias transversales, y demostrar que se puede trabajar en equipo para después enseñarlo.

Se ha señalado del mismo modo como una propuesta de mejora de este indicador el compromiso que se requiere por parte del profesorado-siete referencias, 
19\%-. Estas ideas han sido señaladas especialmente por los grupos formados por estudiantes y por maestros jubilados: Y también es muy importante que los profesores se comprometan. (...) Y para esto es muy importante que los primeros que las tengan adquiridas sean los propios profesores (Carlota, alumna).

Otra de las propuestas con mayor número de referencias -seis referencias, 16\%- es el nuevo paradigma que se requiere para aplicar este modelo. Esta propuesta proviene del grupo de maestros jubilados y sitúan las necesidades del alumnado como aspecto prioritario para este nuevo enfoque de la educación:

Yo veo que lo fundamental es para qué estamos educando. ¿Qué pretendemos de ese alumnado? ¿o qué le vamos a aportar? Porque en un principio la educación sería algo que les sirviese para la vida, y realmente, como estaban hablando ellos, está tan compartimentada que realmente no les sirve (Pablo, maestro jubilado).

En el indicador 212 Propuestas de competencias se han incluido todos los argumentos que tienen que ver con la identificación de nuevas competencias o con la modificación de las ya existentes, para un futuro modelo de competencias en el Grado de Magisterio en educación primaria. Hay 16 referencias codificadas en este indicador. Entre las nuevas propuestas de competencias los participantes destacan la inteligencia emocional -seis referencias, 38\%-, aprender a aprender -cinco referencias, 31\%-y la negociación de conflictos -dos referencias, 13\%-.

Por otro lado, en el indicador Aplicación propuestas -indicador 221- se incluyeron aquellos argumentos o ideas que tuvieron que ver con estrategias de actuación para el desarrollo y evaluación por competencias de los alumnos. Se han registrado 36 referencias. Una de las propuestas con mayor número de referencias -10 referencias, $28 \%$ del indicador- fue la creación de proyectos que vinculen a la universidad con los centros escolares. Esta propuesta se concreta en acciones puntuales y proyectos de larga duración, en los cuales, la colaboración y la interacción con los centros escolares sea permanente. Por ejemplo, Ana (maestra en activo) explica que: Sigo insistiendo en que un programa competencial tiene que ser un programa obligatorio de ida y vuelta, de la facultad a la escuela y de la escuela a 
la facultad.

Otra de las propuestas más numerosas -ocho referencias, $22 \%$ - es la que insiste en la concreción de las competencias: Me refiero que habría que desglosar la competencia para desarrollarla y evaluarla mejor (Daniel, maestro en activo). También relacionada con acciones concretas está la propuesta que establece la necesidad de vincular cada competencia con las asignaturas del plan de estudios del grado de maestro de primaria -cinco referencias, 14\%-.

Con cinco referencias se sitúa la propuesta de incluir trabajos interdisciplinares que puedan desarrollar el modelo por competencias:

A lo mejor se podría plantear algún trabajo interdisciplinar en cada curso para poder trabajarlas. Yo por ejemplo que vengo de formación profesional, se les plantea como una vez al mes un reto que todos los profesores diseñan juntos. Y cada profesor aporta a ese reto una parte de su asignatura. Entonces podría ser como un modelo más práctico (Honorio, maestro en activo).

El resto de propuestas están vinculadas con las prácticas escolares que realizan los estudiantes del grado de maestro de primaria. La más referenciada -cinco referencias- es la que establece aumentar el número de horas de las asignaturas de prácticas escolares, ya que, nosotros nos hemos formado como maestros trabajando, así es como nos hemos hecho competentes, pues ellos deberían adquirirla con práctica (Mar, maestra en activo).

En el indicador 222 Secuenciación de las competencias, se han incluido todos los argumentos tanto positivos como negativos sobre una posible articulación de las competencias. A partir de los resultados aportados en dicho indicador se han obtenido seis referencias -67\%-que confirman dicha secuenciación según los cursos que conforman el grado de maestro de primaria. Por el contrario, tres referencias que opinan de la no secuenciación:

Yo no tengo tan claro que se tengan que secuenciar. En primaria las competencias se trabajan en todos los cursos y en todas las asignaturas. Las posibles competencias que hemos estado trabajando aquí yo creo que todas se pueden trabajar de una forma o de otra en todas 
las asignaturas y momentos (Antonia, maestra en activo).

\section{DISCUSIÓN}

La necesidad de que aparezca un aprendizaje basado en competencias viene ya vinculado a la Declaración de Bolonia en 1999. En los últimos años, se han realizado progresos, pero todavía falta un gran camino por recorrer para llegar a modelos de formación integral de los futuros maestros. Es un momento excelente para actualizar la educación superior con cambios significativos a las nuevas necesidades sociales (Zabalza, 2012).

Una de las dimensiones a estudiar supone detectar problemas que dificulten el desarrollo y evaluación de las competencias transversales, teniendo en cuenta la identificación de elementos positivos y negativos del actual modelo competencial, las competencias transversales consideradas como prioritarias y aplicación de dicho modelo (Almerich et al., 2020).

Con respecto al primer objetivo -analizar la detección de problemas percibidos-, los resultados también se han identificado elementos negativos que deducen una mala y/o escasa práctica actual en relación con este modelo. Numerosos autores (entre otros, Bermúdez et al., 2012; Bolarin-Martínez, 2015; Marko et al., 2018; Torrego y Ruiz, 2011) destacan la importancia de que una competencia pueda ser abordada parcialmente por varias asignaturas con distinto nivel de profundidad. La coordinación docente adquiere un papel clave en el EEES, cuestionando el modelo tradicional que compartimenta las asignaturas (López et al., 2018).

Otro elemento vinculado con la detección de los problemas tiene que ver con la falta de cercanía a la realidad que aporta el modelo competencial actual. Autores como Villa y Poblete (2011) y Calatayud (2019) destacan que evaluar competencias requiere siempre evaluar su aplicación en situaciones reales y en contextos también reales. Uno de los grandes desafíos que destacan es hacer aprender más que enseñar, y para ello invitan a priorizar los recursos ante los saberes, a estimular el aprendizaje a través de situaciones-problema, incorporar retos o desafíos como estrategia educativa, negociar proyectos con los estudiantes y diseñar nuevos con- 
tratos didácticos.

La capacidad de comunicación y de acompañamiento al alumno en sus aprendizajes aparece como una competencia prioritaria en un modelo competencial. Este hecho coincide con el planteamiento realizado desde el Ministerio de Educación, Cultura y Deporte (2015) sobre la formación inicial del profesorado, necesaria para facilitar a los estudiantes la asimilación de conocimientos, habilidades y actitudes, entre las que se destaca la capacidad reflexiva. Conseguir unos futuros maestros competentes en la comunicación y en el acompañamiento al alumnado, ayudará a favorecer una constante autoevaluación, reflexión y buena disposición a cambiar.

En la fase de aplicación por competencias, los informantes del estudio detectan diversas dificultades para su desarrollo. Éstas afectan especialmente al diseño y aplicación de la evaluación (Velasco-Martínez y Tojar, 2018). De acuerdo con Villa y Poblete (2011), lo más importante de la evaluación es la coherencia entre el objeto a evaluar y el procedimiento seleccionado para ello. En este sentido, Gallardo-Fuentes et al. (2020) señalan las dudas que el profesorado puede plantearse a la hora de afrontar la evaluación por competencias, ¿qué y cómo evaluar? En ocasiones se utilizan procedimientos de evaluación que son inadecuados porque se parte de una definición de competencia equivocada o por desconocimiento de las técnicas de evaluación precisas.

La evaluación por competencias requiere la utilización de diversos instrumentos y la implicación de diferentes agentes (Fernández-Ferrer y Forés, 2016). La última de las dificultades encontradas en la aplicación supone la escasa formación del profesorado, es decir, lo que autores determinan como una falta de cultura de colaboración (Zabalza, 2012). La buena enseñanza es una responsabilidad colectiva (Biggs, 2005), de ahí la importancia de conseguir crear una cultura de colaboración en nuestras universidades. Necesitamos adoptar políticas educativas que se fundamenten en la cooperación, a nuevas formas de transformación social que permitan la asunción de diversos puntos de vista (Azorín y Arnaiz, 2018; Montero et al., 2017). 
En relación al segundo objetivo del estudio -analizar las propuestas de mejora-, los resultados justifican la necesidad de implementar cambios y transformaciones asociadas a la convergencia hacia el espacio europeo de educación superior en las condiciones más adecuadas para el futuro laboral, exigiendo una revolución cultural en la formación de competencias (Arrigastaga y Carrera, 2018; Ibarra-Sáiz y Rodríguez-Gómez, 2020). Además, se necesitan profesores competentes con capacidad para desarrollar un modelo competencial e influir en su alumnado de forma positiva, porque esta investigación señala la gran influencia del docente sobre el trabajo por competencias, siendo clave una reflexión crítica sobre su desempeño, sus modos de enseñar y su relación con los alumnos (González et al., 2014).

Los resultados identifican elementos coherentes y viables que no se trabajan en el modelo actual por competencias, como una falta de desarrollo compensado a lo largo de todo el periodo formativo, es decir, se reclama la necesidad de que las competencias transversales aparezcan con la misma importancia que se muestran otros elementos curriculares. En este sentido, autores como Bermúdez et al. (2012) o Bolarin-Martínez y Moreno (2015) plantean que las asignaturas de los primeros cursos han de proporcionar una visión global e introductoria de los estudios, mientras que las de los últimos cursos deben orientar a la reflexión crítica.

Todos los participantes coinciden en incorporar la competencia clave de aprender a aprender a las competencias transversales del Grado en Magisterio en Educación Primaria. De acuerdo con Galvis (2007); Márquez (2009), esta competencia es la base del aprendizaje permanente a lo largo de la vida, sirviendo de soporte para las demás competencias. El objetivo más ambicioso de la educación universitaria sería que el alumnado consiga aprender a aprender, es decir, que sea capaz de asimilar por sí solo, en diversas situaciones y circunstancias, aprendizajes significativos. En este sentido, cobra una gran importancia la exploración, el descubrimiento, la planificación y la regulación como estrategias cognitivas (Conde-Jiménez y Martín-Gutiérrez, 2016).

Por otra parte, se ha señalado como propuesta de mejora la falta de desarro- 
llo y evaluación de las competencias emocionales. De acuerdo con Álvarez (2018), abordar este tipo de competencias supone al docente del siglo XXI un gran reto porque le demanda no solo consolidar sus competencias técnico-disciplinares, sino también su desarrollo humano y competencia emocional. En la actualidad, esta última se presenta como una competencia emergente que permite al profesorado conseguir intervenciones eficaces.

A este respecto es conveniente conocer la clasificación de las competencias emocionales confeccionada por Hué (2013), diferenciando entre aquellas vinculadas con uno mismo, tales como el autoconocimiento, la autoestima, el autocontrol, la adaptación y la toma de decisiones, y por otro lado, las relacionadas con los demás, tales como la empatía, el trabajo grupal, la resolución de conflictos y el liderazgo.

En este sentido, se han encontrado estudios que reflejan en los estudiantes posibles miedos o rechazo al trabajo en grupo, debido a los posibles conflictos que pueda generar en los participantes (García-Merino et al., 2016). Es importante conocer que el significado del aprendizaje es personal, y los estudiantes experimentan la necesidad de llegar a la meta o al reto, siendo la motivación un producto de la buena enseñanza, no el prerrequisito del alumno. Pero el constructivismo se centra principalmente en la naturaleza de las actividades de aprendizaje que realiza el estudiante; donde se observa que el medio -el contexto- es un elemento clave, y los estudiantes pueden elegir un enfoque más profundo o superficial, dependiendo del contexto de la enseñanza. Por lo tanto, nos encontramos con una interacción entre lo personal y lo contextual (Fernández-Ferrer y Forés, 2016).

Otro elemento coherente y significativo que destacan los participantes es la importancia de introducir metodologías activas -aprendizaje basado en retos, en proyectos, cooperativo, basado en el diseño, basado en problemas, basado en el pensamiento, etc.- que fomentan y facilitan el desarrollo de un modelo formativo por competencias transversales, consiguiendo a través de esto que los estudiantes aprendan lo más próximos posible a la realidad educativa (Gargallo, 2016; González et al., 2018; Swartz et al., 2015; Vergara, 2015). También se pueden generar a través de 
estas metodologías activas proyectos que vinculen a la universidad con los centros escolares, donde los alumnos aprendan con otra mirada y les permitan construir una identidad docente rigurosa, fundamentada y reflexionada con las características de la escuela y los progresos del conocimiento pedagógico actuales (Jarauta y Pérez, 2017).

Además, es importante recordar que el objetivo es formar maestros/as reflexivos que les permita convivir, adecuándose a las circunstancias del contexto (Aguado et al., 2017). Por lo tanto, en la fase de aplicación sobre un futuro modelo competencial, los participantes de este estudio destacan la importancia del trabajo interdisciplinar, coincidiendo con Martínez et al. (2016). Incluso, llegan a considerarla como una de las competencias transversales prioritaria, que va más allá del trabajo en equipo, es decir, la capacidad de trabajar en equipo de forma interdisciplinar. De esta forma, conseguiremos acercar a los alumnos a una aplicación más práctica, a contextos reales dentro de las asignaturas. Para ello, se requiere de la negociación y de la flexibilidad de las planificaciones enfocadas desde la interdisciplinariedad, la cual, reduce la brecha entre asignaturas, unificando sus destinos por un fin común (Marko et al., 2018; Ramos, 2005).

A partir del elemento negativo identificado de la coordinación docente, y para conseguir mejorar la comprensión interdisciplinar señalada anteriormente, los participantes de este estudio destacan en la fase de aplicación la importancia de que exista una buena coordinación entre asignaturas, departamentos y profesores. Una condición previa del trabajo por competencias es que la elaboración de los planes de estudio tiene que fundamentarse en una visión en conjunto mediante la coordinación de asignaturas, evitando el solapamiento o la ausencia de contenidos y competencias.

Algunas de las propuestas de desarrollo aportadas en este estudio coinciden con las estrategias planteadas por Zabalza (2012) y reforzadas en el trabajo de (Martínez et al., 2016). Sirvan como ejemplo la necesidad de tener una estructura sistémica que dé unidad y sentido formativo a las competencias integradas en el Grado 
en Magisterio en Educación Primaria.

Toda la información planteada en esta discusión nos hace reflexionar críticamente, como destaca Benayas y Marcén (2019), sobre el tipo de competencias abordadas desde la educación superior para comprometerse con las necesidades de la sociedad, promoviendo la creación y transferencia de conocimiento. Educar es crear libertad, dar posibilidad, hacer, pensar. Por lo tanto, la enseñanza universitaria debería proponer desarrollar una educación permanente más ligada a la vida, apoyando una educación moderna, libre, creadora y solidaria que se fundamente en el diálogo reflexivo y no en la mera transmisión memorística y acrítica del saber.

El reto para la Facultad de Educación es avanzar decididamente hacia proporcionar una base consistente para el desarrollo y evaluación de las competencias transversales, a través de un entorno colaborativo -cultura de colaboración- para seguir mejorando el proceso formativo del alumnado. Por este motivo, debe orientar sus esfuerzos hacia una reflexión crítica de la praxis, que tenga una mayor incidencia en un enfoque por competencias.

Debe de aprovechar las continuas revisiones de las guías docentes y la necesidad de un enfoque interdisciplinar para enfatizar en el desarrollo de las competencias transversales más valoradas -capacidad de comunicar, inteligencia emocional y aprender a aprender-, e integrando elementos transformadores y de calidad como los Objetivos de Desarrollo Sostenible. Un modelo de formación competencial y la contribución del mismo a la Agenda 2030 para el desarrollo sostenible en el contexto universitario exige cambios que sean capaces de emplear enfoques integrados que produzcan resultados transformadores y con transferencia a otros contextos. Necesitamos crear una hoja de ruta y pensar de forma holística, colaborativa e innovadora para preparar a los futuros maestros/as con las competencias y herramientas necesarias para convertirse en agentes de cambio.

\section{REFERENCIAS}

Aguado, D., González, A., Antúnez, M. y De Dios, T. (2017). Evaluación de Competencias Transversales en Universitarios. Propiedades Psicométricas Iniciales del 
Cuestionario de Competencias Transversales. REICE, Revista Iberoamericana sobre Calidad, Eficacia y Cambio en Educación, 15(2), 129-152. https:// doi.org/10.15366/reice2017.15.2.007

Almerich, G., Suárez-Rodríguez, J., Díaz-García, I. y Orellana, N. (2020). Estructura de las competencias del siglo XXI en alumnado del ámbito educativo. Factores personales influyentes. Educación XX1, 23(1), 45-74. https://doi.org/10.5944/ educxx1.23853

Álvarez, E. (2018). La competencia emocional como reto en la formación y actualización docente. Reflexiones a partir de un estudio de casos en educación básica en México. Controversias y Concurrencias Latinoamericanas, 10, 1-17. Arrigastaga, E. y Carrera, X. (2018). Necesidades a futuro y situación actual de las competencias en Educación Superior en el contexto de España. RIDU, Revista Digital de Investigación en Docencia Universitaria, 12(2), 35-58. https://doi.org/10.19083/ridu.2018.731

Azorín, C. y Arnaiz, P. (2018). Redes de colaboración en educación. Nuevas formas de participación y transformación social. Profesorado. Revista de currículum y formación del profesorado, 22(2), 1-6. https://doi.org/10.30827/profesorado.v22i2.7846

Bardin, L. (2002). Análisis de contenido. Akal.

Benayas, J. y Marcén, C. (Coords.) (2019). Hacia una educación para la sostenibilidad. Centro Nacional de Educación Ambiental.

Bermúdez, A., García-Varea, I., López, M. T., Montero, F., de la Ossa, L., Puerta, J. M., Rojo, T. y Sánchez, J. L. (2012). Una experiencia de coordinación docente en Ingeniería Informática. ReVisión, 5(2), 17-29.

Bicocca, M. (2018). Competencias, capacidades y Educación Superior. Repensando el desarrollo humano en la Universidad. Estudios sobre Educación, 34, 2946. https://doi.org/10.15581/004.34.29-46

Biggs, J. (2005). Calidad del aprendizaje universitario. Narcea. 
Bolarin-Martínez, M. J. y Moreno, M. A. (2015). La coordinación docente en la universidad: retos y problemas a partir de Bolonia. Profesorado. Revista de Currículum y Formación de Profesorado, 19(2), 319-332.

Bolívar, A. (2011). Las competencias básicas para la vida más transversales. Buenas prácticas para su tratamiento en el centro educativo y en el aula. USAID y Ministerio de Educación.

Calatayud, M. A. (2019). Una oportunidad para avanzar hacia la evaluación auténtica en Educación Física. Retos, 36, 259-265. https://doi.org/10.47197/retos. v36i36.67540

Conde-Jiménez, J. y Martín-Gutiérrez, A. (2016). Potencialidades y necesidades de mejora en la formación de profesores noveles universitarios. Revista Electrónica de Investigación Educativa, 18(1), 140-152.

Fernández-Ferrer, M. y Forés, A. (2016). Evaluación del desarrollo competencial en la educación superior. La perspectiva del profesorado universitario. Educar, 54(2), 391-410.

Fraile, A. (2006). El sistema universitario europeo como modelo posible para la educación superior latinoamericana. REDIE, Revista Electrónica de Investigación Educativa, 8(1), 1-15.

Galvis, R. V. (2007). De un perfil docente tradicional a un perfil docente basado en competencias. Acción Pedagógica, 16, 48-57.

Gallardo-Fuentes, F. J., López-Pastor, V. M. y Carter-Thuillier, B. I. (2020). Ventajas e Inconvenientes de la Evaluación Formativa, y su Influencia en la Autopercepción de Competencias en alumnado de Formación Inicial del Profesorado en Educación Física. Retos, 38, 417-424. https://doi.org/10.47197/retos. v38i38.75540

García-Merino, J. D., Urionabarrenetxea, S. y Bañales-Mallo, A. (2016). Cambios en metodologías docente y de evaluación: ¿mejoran el rendimiento del alumnado universitario? Revista Electrónica de Investigación Educativa, 18(3), 1-18. 
Gargallo, B. (Coord.) (2016). Enseñanza centrada en el aprendizaje y diseño por competencias en la universidad. Fundamentación, procedimientos y evidencias de aplicación e investigación. Tirant Humanidades.

González, J. M., Arquero, J. L. y Hassall, T. (2014). Consolidación de la formación por competencias en la universidad española: estudio de un caso. Educación XXI, 17(2), 145-168. https://doi.org/10.5944/educxx1.17.2.11483

González, N., Pérez, J. y Martínez, M. (2018). Desarrollo de competencias transversales en la Universidad de Murcia: fortalezas, debilidades y propuestas de mejora. RIDU, Revista Digital de Investigación en Docencia Universitaria, 12(2), 88-113. http://dx.doi.org/10.19083/ridu.2018.727

Hué, C. (2013). Una experiencia de formación en competencias emocionales del profesorado universitario. Revista d'Innovació Docent Universitària, 5, 4261. https://doi.org/10.1344/105.000001865

Ibarra-Sáiz, M. S. y Rodríguez-Gómez, G. (2020). Aprendiendo a evaluar para aprender en la educación superior. Revista Iberoamericana de Evaluación Educativa, 13(1), 5-8.

Jarauta, B. y Pérez, M. J. (2017). La construcción de la identidad profesional del maestro de Primaria durante su formación inicial. El caso de la Universidad de Barcelona. Profesorado. Revista de currículum y formación del profesorado, 21(1), 103-122. https://doi.org/10.30827/profesorado.v21i1.10354

Jiménez, Y. I., Gutiérrez, J. J. y Hernández, J. (2019). Logros y desafíos en la formación de competencias transversales por áreas de conocimiento en la Educación Superior del Instituto Politécnico Nacional (México). Formación Universitaria, 12(3), 91-100. http://dx.doi.org/10.4067/S0718-50062019000300091

López, M. C., León, M. J. y Pérez, P. (2018). El enfoque por competencias en el contexto universitario español. La visión del profesorado. Revista de Investigación Educativa, 36(2), 529-545. https://doi.org/10.6018/rie.36.2.314351 
Marko, I., Pikabea, I., Altuna, J., Eizagirre, A. y Pérez-Sostoa, V. (2018). Propuesta para el desarrollo de competencias transversales en el Grado de Pedagogía. Un estudio de caso. Revista Complutense de Educación, 30(2), 381-398. https:// doi.org/10.5209/RCED.57490

Márquez, A. C. (2009). La Formación Inicial para el nuevo perfil del Docente de Secundaria. Relación entre la teoría y la práctica [Tesis Doctoral]. Universidad de Málaga (España). http://www.biblioteca.uma.es/bbldoc/tesisuma/17676356.pdf

Martínez, P. y González, N. (2019). El dominio de las competencias transversales en Educación Superior en diferentes contextos formativos. Educação e Pesquisa, 45, e188436. https://doi.org/10.1590/s1678-4634201945188436

Martínez, P., González, N. y González, C. (2016). Percepción de competencias y plan de mejora para los títulos de grado en Educación: la mirada de los estudiantes. Estudios Pedagógicos, 42(3), 277-292. http://dx.doi.org/10.4067/ S0718-07052016000400015

Ministerio de Educación, Cultura y Deporte (España). Consejo Escolar del Estado (2015). El profesorado del siglo XXI. Encuentro de Consejos Escolares Autonómicos y del Estado. https://www.educacionyfp.gob.es/educacion/mc/ cee/actuaciones/junta-de-participacion/21encuentro.html

Montero, L., Martínez, E. y Colén, M. (2017). Desarrollo del conocimiento profesional en la formación inicial de maestros. Profesorado. Revista de currículum y formación del profesorado, 27(1), 1-16.

Montero, M. (2010). El Proceso de Bolonia y las nuevas competencias. Tejuelo, 9, 1937.

Morales, J. A. y Morales, F. M. (2018). Evaluación de competencias genéricas y socioemocionales por parte de universitarios. European Journal of Child Development, Education and Psychopathology, 6(2), 97-105. https://doi. org/10.30552/ejpad.v6i2.72 
Onieva, J. C. (2018). Scrum como estrategia para el aprendizaje colaborativo a través de proyectos. Propuesta didáctica para su implementación en el aula universitaria. Profesorado. Revista de currículum y formación del profesorado, 22(2), 509-527. https://doi.org/10.30827/profesorado.v22i2.7735

Pinos, M. (2016). Evaluación de un programa educativo de competencias, educación en valores y motivación escolar [Tesis doctoral]. Universidad de Zaragoza (España). https://zaguan.unizar.es/record/57707/files/TUZ_0957_pinos_evaluacion.pdf

Ramos, S. L. (2005). El desarrollo de las competencias didácticas: un reto en la formación inicial de los futuros docentes de primaria. Educar, 35, 49-60.

Stufflebeam, D. L. y Shinkfield, A. J. (1987). Evaluación sistemática. Guía teórica y práctica. Paidós.

Swartz, R. J., Costa, A. L., Beyer, B. K., Reagan, R. y Kallick, B. (2015). El aprendizaje basado en el pensamiento. Cómo desarrollar en los alumnos las competencias del siglo XXI. SM.

Torrego, L. y Ruiz, C. (2011). La coordinación docente en la implantación de los títulos de Grado. Revista Electrónica Interuniversitaria de Formación del Profesorado, 12(2), 121-144.

UNESCO. (2017). Educación para los objetivos de desarrollo sostenible. Objetivos de aprendizaje. Autor. https://unesdoc.unesco.org/ark:/48223/ pf0000252423

Velasco-Martínez, L. C. y Tójar, J. C. (2018). Uso de rúbricas en educación superior y evaluación de competencias. Profesorado. Revista de currículum y formación del profesorado, 22(3), 183-208. https://doi.org/10.30827/profesorado. v22i3.7998

Vergara, J. J. (2015). Aprendo porque quiero. El aprendizaje basado en proyectos $(A B P)$, paso a paso. SM.

Villa, A. y Poblete, M. (2011). Evaluación de competencias genéricas: principios, oportunidades y limitaciones. Bordón, 63(1), 147-170. 
Zabalza, M. A. (2012). Las competencias en la formación del profesorado: de la teoría a las propuestas prácticas. Tendencias pedagógicas, 20, 5-32.

\section{Fecha de recepción 29 de junio de 2021}

Fecha de aceptación 9 de septiembre de 2021

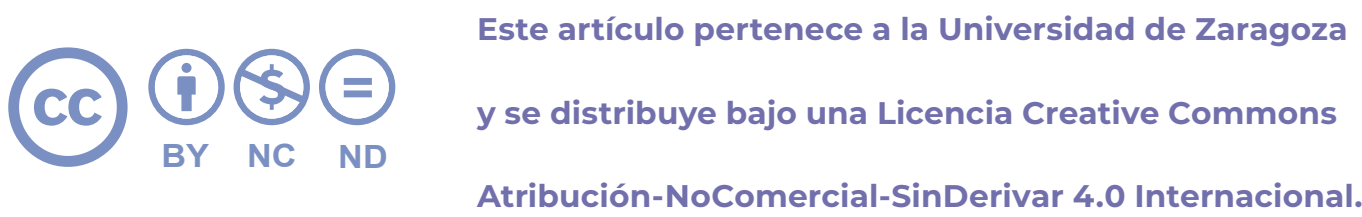

Eres libre de compartir copiar y redistribuir el material en cualquier medio o formato -

Bajo las condiciones siguientes:

- Reconocimiento de la autoría, ya incluida en esta diapositiva.

- NoComercial — no se puede utilizar el material para una finalidad comercial.

- SinObraDerivada - Sin remezclar, transformar o crear a partir del material. 